九州大学学術情報リポジトリ

Kyushu University Institutional Repository

Micro-segregation induced by bulky-head Lipids: Formation of characteristic patterns in a giant vesicle

柳澤，実穂

http://hdl. hand le. net/2324/21699

出版情報：(8)，pp.488-495，2012

バージョン:

権利関係 : 


\title{
Micro-segregation induced by bulky-head lipids: formation of characteristic patterns in a giant vesicle
}

\author{
Miho Yanagisawa, ${ }^{* a}$ Naofumi Shimokawa, ${ }^{b}$ Masatoshi Ichikawa ${ }^{c}$ and Kenichi Yoshikawa ${ }^{* c}$ \\ Received 21st July 2011, Accepted 28th September 2011 \\ DOI: $10.1039 / \mathrm{c} 1 \mathrm{sm} 06381 \mathrm{~b}$
}

Bulky-head lipids, such as glycolipids, play indispensable roles in cellular membranes. However, little is known about the effects that bulky-head lipids have on the physicochemical properties of the membrane. In this study, we examined the effects of a giant vesicle containing poly(ethylene glycol)conjugated cholesterol (PEG-Chol), as a model of natural bulky-head lipids, on phase separation in the membrane. We used a lipid combination that included the saturated phospholipid DPPC, the unsaturated phospholipid DOPC and Chol, which is known to cause phase separation into two liquid phases. This phase separation is classified as a first-order phase transition under the criterion of Landau, and thus micro-domains tend to show coarse-graining up to a global pair of domains, i.e., mono-domains, so as to minimize interfacial instability. In contrast to such coarsening in the ternary system, we show here the generation of stable micro-domains in the presence of PEG-Chol above a critical composition. The transition from global- to micro-segregation is interpreted theoretically in terms of the competition between two physical effects, i.e., steric repulsive interaction between the bulky-head groups of PEG-Chol and the cost in line energy along the domain boundaries. Interestingly, among the micro-domain structures, a network pattern of domains appears as an intermediate state in which small domains are connected to each other. We examined the stability of the network pattern under local heating using a focused laser, and confirmed self-recovery of the pattern. Based on these observations, natural bulky-head lipids in cells may also stabilize the domain structure like a lipid raft.

\section{Introduction}

According to the "lipid raft" hypothesis, biomembranes are not homogeneous mixtures of lipids, but rather form nano-domain structures that are rich in saturated lipids and cholesterol (Chol). ${ }^{1}$ These domains, which are called lipid rafts, are thought to play important roles in many membrane-associated events such as signal transduction and intracellular trafficking, and have attracted attention for decades. ${ }^{2}$ Subsequent studies have suggested that saturated lipids and glycosyl-phosphatidylinositol (GPI)-anchored proteins integrate, and form raft domains with the aid of Chol. ${ }^{3-5}$

Such Chol-mediated domains are often speculated to arise from the tendency for lipids in membranes containing Chol to separate into coexisting liquid phases. Indeed, one of the simplest model biomembranes, ternary giant unilamellar vesicles (GUVs) composed of dipalmitoyl-phosphatidylcholine (DPPC), dioleoylphosphatidylcholine (DOPC) and Chol, show phase

${ }^{a}$ Department of Physics, Graduate School of Sciences, Kyushu University, Fukuoka,812-8581, Japan. E-mail: yanagisawa@phys.kyushu-u.ac.jp ${ }^{b}$ Institute of Industrial Science, The University of Tokyo, Tokyo, 153-8505, Japan

'Department of Physics, Graduate School of Science, Kyoto University, Kyoto, 606-8501, Japan. E-mail: yoshikaw@scphys.kyoto-u.ac.jp separation into a liquid-ordered (Lo) phase that is rich in DPPC and a liquid-disordered (Ld) phase that is rich in DOPC. ${ }^{6-12}$ Numerous studies have been performed with different species of lipids to clarify the structure of rafts, and the results have been reported with regard to phase diagrams, ${ }^{\mathbf{7 , 9 , 1 3 , 1 4}}$ domain-coarsening, ${ }^{7,10,15}$ etc. However, real cells contain not only bare lipids but also a large number of bulky glycolipids. In the case of GPIanchored protein CD59, the cross-sectional area is more than 9 times greater than that of lipids. ${ }^{16}$ Therefore, such bulky-head lipids may play a main role on the formation of nano-domains in cells.

In this study, we used poly(ethylene glycol)-conjugated cholesterol (PEG-Chol) as a model of glycolipids to elucidate the effects of bulky-head lipids on phase separation. The physical nature of lipid bilayers containing PEG-lipids has been studied extensively. For example, PEG-lipids in a PC membrane decrease the main gel-to-liquid-crystal transition temperature of lipids, ${ }^{17,18}$ increase the bending rigidity ${ }^{19-21}$ and induce a finite spontaneous curvature. ${ }^{22}$ On the other hand, there have been no studies on the effects of PEG-lipids on phase separation in GUVs. Therefore, we first prepared a basic ternary GUV composed of DPPC, DOPC and Chol, which has been investigated experimentally $y^{7,8,10}$ and theoretically. ${ }^{23,24} \mathrm{Next}$, we gradually replaced Chol in the ternary GUVs with PEG-Chol. By 
comparing the domain patterns observed with and without PEG-Chol, we could elucidate the effects of PEG-Chol on phase separation. This study may shed light on the intrinsic mechanism of the stabilization of nano-domain structures in cells containing large numbers of bulky-head lipids and proteins.

\section{Experimental}

\section{Materials}

The lipids 1,2-dipalmitoyl-sn-glycero-3-phosphocholine (DPPC) and 1,2-dioleoyl-sn-glycero-3-phosphatidylcholine (DOPC) were obtained from Wako Pure Chemical Industries (Osaka, Japan), and cholesterol (Chol) was from Sigma (St Louis, MO). PEGconjugated Chol (PEG-Chol) with an average molecular weight $\left(M_{\mathrm{w}}\right)$ of 600,1000 , or 2000 was purchased from Sigma or Nihon Yushi (Tokyo, Japan). The chemical structure of PEG600-Chol is shown in Fig. 1. The radius of gyration of PEG in water is roughly $R_{\mathrm{G}}=0.6 \mathrm{~nm}\left(M_{\mathrm{w}}=600\right), 0.9 \mathrm{~nm}\left(M_{\mathrm{w}}=1000\right)$ and $1.3 \mathrm{~nm}\left(M_{\mathrm{w}}=2000\right)$ based on the experimentally determined relationship $R_{\mathrm{G}}=0.01078 M_{\mathrm{PEO}}{ }^{0.635} \mathrm{~nm} .{ }^{25}$ The persistence length of PEG is $\sim 0.9 \mathrm{~nm}^{26}$ The cross-sectional areas of DPPC, DOPC and Chol are approximately $0.5,0.72$ and $0.37 \mathrm{~nm}^{2}$, respectively. ${ }^{27,28}$ The fluorescent lipids 1,2 dioleolyl-sn-glycero-3-phosphoethanolamine- $N$-(lissamine-rhodamine-B-sulfonyl) (Rho-PE) and 1-acyl-2[6-((7-nitro-2-1,3-benzoxadiazol-4-yl)amino)hexanoyl]-sn-glycero-3phosphoethanolamine (NBD-PE) were purchased from Avanti Polar Lipids (Alabaster, AL). The former dye partitions into the DOPC-rich Ld phase and gives a red color, whereas the latter preferentially partitions into the DPPC-rich Lo phase and gives a green color. All lipids were used without further purification and stored in chloroform at $-20{ }^{\circ} \mathrm{C}$.

\section{Methods and observations}

We prepared ternary GUVs by the gentle hydration method. ${ }^{10}$ First, we dissolved lipids in chloroform $(10 \mathrm{mM})$. For a fluorescent observation, Rho-PE and NBD-PE were added at 0.4 and $0.2 \mathrm{~mol} \%$ of lipids, respectively. The solvent was evaporated in a stream of nitrogen gas, and the obtained lipid film was kept under vacuum overnight to completely remove the remaining solvent. To keep the temperature certainly above the phase separation point $<35^{\circ} \mathrm{C}$, the dried lipid film was pre-warmed at $60{ }^{\circ} \mathrm{C}$, and then hydrated with $1 \mathrm{ml}$ of pure water at $60{ }^{\circ} \mathrm{C}$. During the hydration process, the lipid films spontaneously form GUVs with diameters of 5-30 $\mu \mathrm{m}$. Phase separation in GUVs was observed using a confocal fluorescence microscope (LSM510, Carl Zeiss, Jena, Germany). Rho-PE was excited with a $\mathrm{He}-\mathrm{Ne}$ laser $(543 \mathrm{~nm})$ and fluorescence was detected through a 560-615 nm band-pass filter (Chroma, Rockingham, VT).

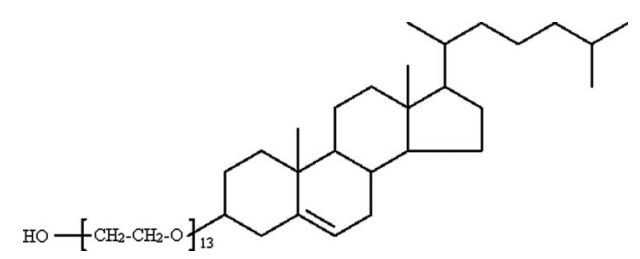

Fig. 1 Chemical structure of PEG600-cholesterol.
NBD-PE was excited by an Ar laser (488 nm) and fluorescence was detected through a 505-530 nm band-pass filter (Chroma). To avoid photo-oxidation, we minimized exposure to light.

To investigate the thermal and mechanical stabilities of the domains, an infrared laser (1064 nm; YML-10, IPG Photonics, Oxford, MA) in continuous wave mode was used. The laser beam was converged and manipulated on the focal plane by an optical trapping system (Sigma-Koki, Tokyo, Japan) with a fluorescent microscope (TE2000, Nikon, Tokyo, Japan). Each laser had a source power of approximately $2 \mathrm{~W}$, which corresponds to a temperature rise of $20-30{ }^{\circ} \mathrm{C}$ at the focus and a rise of $\sim 10{ }^{\circ} \mathrm{C}$ within $\mathrm{a} \sim 10 \mu \mathrm{m}$ radius around the focus.

\section{Results}

Ternary GUVs composed of the saturated lipid DPPC, the unsaturated lipid DOPC and Chol show three distinct phases, i.e., solid-liquid coexistence phase, liquid-liquid coexistence phase and a homogeneous phase, based on the lipid composition and temperature. ${ }^{\mathbf{8} 10}$ To investigate the effects of PEG-lipids on phase separation, we prepared three different systems described below, and examined the domain patterns in terms of two parameters: the area fraction of the DPPC-rich ordered (Lo) phase, $\phi_{\mathrm{o}}$, and the domain boundary length, $l$.

First, as a basic system, we prepared ternary GUVs consisting of DPPC/DOPC $=1: 1$ plus various mole fractions of Chol, $m_{\text {chol }}\left(0 \leq m_{\text {chol }} \leq 0.5\right)$, and observed the formation of domain patterns with an increase in $m_{\text {chol }}$. Next, we gradually added PEG-Chol to the basic ternary system. The ratio of PEGChol to total Chol, $X_{\mathrm{PEG}}=m_{\mathrm{PEG}} /\left(m_{\mathrm{PEG}}+m_{\mathrm{chol}}\right)$, was increased from 0 to $1\left(0 \leq X_{\mathrm{PEG}} \leq 1\right)$, where $m_{\mathrm{PEG}}$ is the mole fraction of PEG-Chol, and the total mole fraction of Chol, $m_{\mathrm{PEG}}+$ $m_{\text {chol, }}$, was fixed at 0.33 (i.e., DPPC/DOPC/(Chol and PEGChol) $=1: 1: 1)$. Finally, we prepared other ternary vesicles consisting of DPPC/DOPC $=1: 1$ plus PEG-Chol, and increased $m_{\mathrm{PEG}}$ from 0 to $0.5\left(0 \leq m_{\mathrm{PEG}} \leq 0.5\right)$. By comparing the domain patterns observed in GUVs with and without PEGChol, we could elucidate the effects of PEG-Chol on phase separation.

\section{Ternary vesicles: DPPC/DOPC $=1: 1$ plus Chol}

Ternary GUVs composed of DPPC/DOPC $=1: 1$ plus Chol cause phase separation into coexisting phases below a transition temperature. Fig. 2a shows an example of phase-separated vesicles with micro-domains, where bright and dark regions are DOPC-rich and DPPC-rich phases, respectively. For binary vesicles without Chol, DPPC solid domains were observed as black islands floating on a white sea of liquid DOPC. The observed solid domains had noncircular shapes (far-left image in Fig. 2a). When the two solid domains contacted each other, they did not merge. On the other hand, ternary vesicles containing Chol $\left(0.1<m_{\text {chol }}<0.5\right)$ separated into two liquid phases, i.e., a DPPC-rich liquid-ordered (Lo) phase and a DOPC-rich liquiddisordered (Ld) phase. For vesicles with $m_{\text {chol }} 0.2-0.3$, circular Lo domains appeared. Above $m_{\text {chol }} \approx 0.35$, the major phase was reversed, and Ld domains were formed. With the addition of more Chol $\left(m_{\text {chol }} \geq 0.5\right)$, phase separation was not observed. 
a

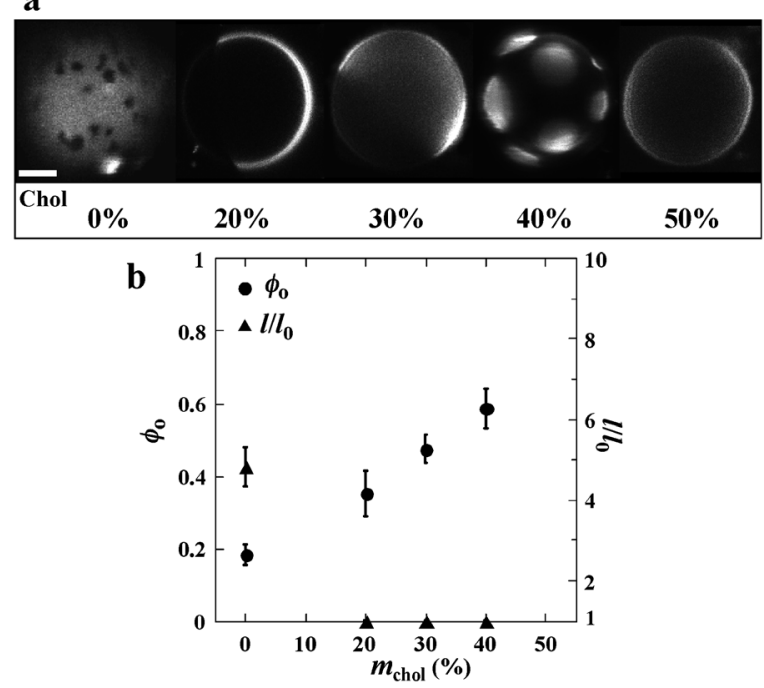

Fig. 2 Phase separation for ternary vesicles composed of DPPC/DOPC $=1: 1$ plus Chol. (a) The micrograph shows phase-separated vesicles with different mole fractions of Chol, $m_{\text {chol }}$, where the dark and bright regions are a DPPC-rich ordered phase and a DOPC-rich disordered phase, respectively. Scale bar is $5 \mu \mathrm{m}$. (b) The area fraction of the ordered phase, $\phi_{\mathrm{o}}$ (circles), and the boundary length normalized by the minimum length of a domain with the same area, $l / l_{0}$ (triangles), are plotted against $m_{\text {chol }}$.

For vesicles with coexisting Lo and Ld phases, the line energy along the domain boundaries plays an important role in regulating the domain shape and the number of domains. ${ }^{10,29}$ To avoid the cost associated with a large line energy, both the Lo and $\mathrm{Ld}$ domains maintain circular shapes, and coarse-grain up to a large mono-domain with a minimum boundary length. Although some vesicles had multi-domains due to trapped coarsening with budding deformation (see Chol $40 \%$ in Fig. 2a), ${ }^{10}$ most had a mono-domain. These observations are consistent with previous reports. ${ }^{6,8,10}$ We selected 30 vesicles with a mono-domain, and calibrated the area fraction of the DPPC-rich ordered phase, $\phi_{\mathrm{o}}$, and its boundary length normalized by the minimum boundary length of a circular domain with the same area, $l / l_{0}$. Fig. $2 \mathrm{~b}$ shows the relationship of $\phi_{\mathrm{o}}$ and $l / l_{0}$ with the mole fraction of Chol, $m_{\mathrm{chol}}$. $\phi_{\mathrm{o}}$ gradually increased with an increase in $m_{\text {chol }}{ }^{12}$ On the other hand, $l / l_{0}$ has a value of $\sim 1$ except for $m_{\text {chol }}=0$, which means that the vesicles show a spherical mono-domain, unlike scattered solid domains in vesicles without Chol, i.e., $m_{\mathrm{chol}}=0$.

\section{Four-component vesicles with bulky-head lipids}

DPPC/DOPC = 1: 1 plus Chol and PEG-Chol. To investigate the effects of PEG-Chol on phase separation, we prepared fourcomponent GUVs with DPPC/DOPC $=1: 1$ plus Chol and PEG-Chol. The ratio of PEG-Chol to total Chol, $X_{\mathrm{PEG}}=m_{\mathrm{PEG}} /$ $\left(m_{\mathrm{PEG}}+m_{\text {chol }}\right)$, was increased from 0 to $1\left(0 \leq X_{\mathrm{PEG}} \leq 1\right)$, where the total mole fraction of Chol, $m_{\mathrm{PEG}}+m_{\mathrm{chol}}$, was fixed at $\sim 0.33$. If PEG-Chol behaves like Chol, the domain pattern should be the same as in the Chol system; i.e., DPPC/DOPC $=1: 1$ plus Chol $33 \%$ (see $m_{\text {chol }} \approx 0.3$ in Fig. 2). However, the domain pattern changed dramatically with the addition of PEG-Chol.
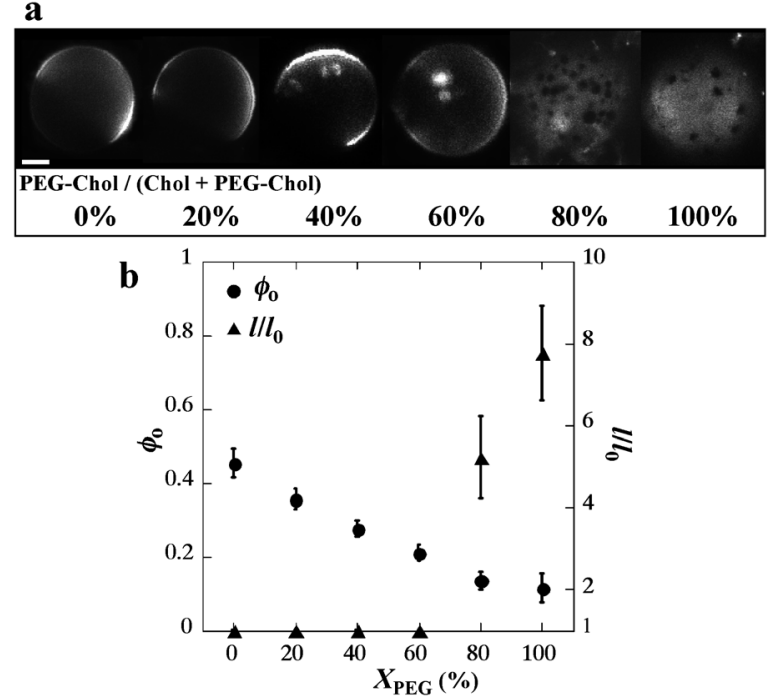

Fig. 3 Phase separation for four-component vesicles composed of DPPC/DOPC/(total Chol; Chol and PEG-Chol) $=1: 1: 1$. (a) The images show a change in the domain size with an increase in the mole ratio of PEG-Chol to total Chol, $X_{\mathrm{PEG}}$. The dark and bright regions are a DPPC-rich ordered phase, and a DOPC-rich disordered phase, respectively. Scale bar is $5 \mu \mathrm{m}$. (b) The area fraction of the ordered phase, $\phi_{\mathrm{o}}$ (circles), and the normalized boundary length, $l / l_{0}$ (triangles), are plotted against $X_{\mathrm{PEG}}$.

Fig. 3a shows the observed domain patterns on vesicles containing PEG-Chol, where bright and dark regions are the DOPCrich disordered phase and DPPC-rich ordered phase, respectively. At a concentration of PEG-Chol $X_{\mathrm{PEG}}<0.8$, the size of a DPPC-rich domain gradually decreased with an increase in $X_{\mathrm{PEG}}$, while the vesicle maintained a mono-domain structure. On the other hand, above $X_{\mathrm{PEG}} \geq 0.8$, the domain structure was transformed from a mono-domain to small scattered domains. Since the scattered domains did not coalesce together and were maintained overnight, they might represent an equilibrium state. Therefore, we concluded that a transition from global- to microsegregation is induced in the presence of PEG-Chol above a critical concentration of $X_{\mathrm{PEG}} * \approx 0.8$.

To examine these phenomena, the area fraction of the ordered phase, $\phi_{\mathrm{o}}$, and the boundary length normalized by the minimum boundary length of a domain with the same area, $l / l_{0}$, were calibrated from 30 vesicles and plotted against $X_{\mathrm{PEG}}$ in Fig. $3 \mathrm{~b}$. With an increase in $X_{\mathrm{PEG}}$ from $X_{\mathrm{PEG}}=0, \phi_{\mathrm{o}}$ monotonically decreases from $\sim 0.5$ to $\sim 0.1$ for $X_{\mathrm{PEG}}<0.8$, while $l / l_{0}$ remains at a value of 1 . On the other hand, for $X_{\mathrm{PEG}} \geq 0.8, \phi_{\mathrm{o}}$ maintains a value of $\sim 0.1$, and $l / l_{0}$ dramatically increases from 1 to $\sim 8$ accompanied by a transition from a mono-domain to small scattered domains. We explain this transition theoretically below.

\section{Ternary vesicles with bulky-head lipids}

DPPC/DOPC = 1: 1 plus PEG-Chol. We completely replaced Chol with PEG-Chol, and prepared other ternary GUVs composed of DPPC/DOPC $=1: 1$ plus PEG-Chol, $m_{\mathrm{PEG}}=0$ 0.5 . The obtained micrographs of phase-separated vesicles are shown in Fig. 4a, where bright and dark regions are the 


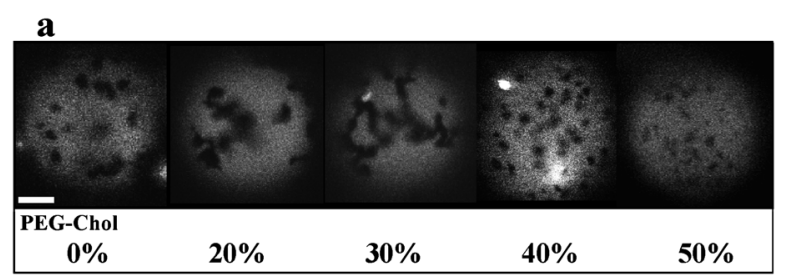

b

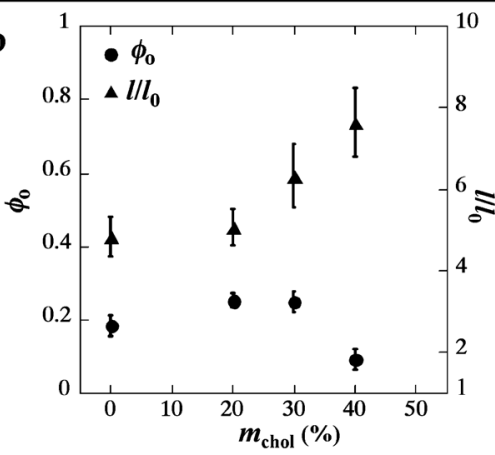

Fig. 4 Phase separation on ternary vesicles composed of DPPC/DOPC $=1: 1$ plus PEG-Chol. (a) Micrographs show the transition from network to scattered domains with an increase in molar ratio of PEGChol, $m_{\mathrm{PEG}}$. The bright and dark regions are DOPC-rich and DPPC-rich phases, respectively. Scale bar is $5 \mu \mathrm{m}$. (b) The area fraction of the DPPCrich ordered phase, $\phi_{\mathrm{o}}$ (circles), and the normalized boundary length, $l / l_{0}$ (triangles), are plotted against $m_{\mathrm{PEG}}$.

DOPC-rich disordered phase and DPPC-rich ordered phase, respectively. In vesicles with large $m_{\mathrm{PEG}}>0.3$, scattered domains of the ordered phase were observed, like a vesicle with $m_{\mathrm{PEG}} \approx$ 0.33 (far-right image in Fig. 3a). Interestingly, the other domain pattern was observed in vesicles with $0<m_{\mathrm{PEG}} \leq 0.3$, where small domains were connected to each other. We refer to this as a "network domain" hereafter. The critical concentration of PEG-Chol $m_{\mathrm{PEG}}{ }^{*}$ at the transition point was $\sim 0.3$.

We measured the area fraction of the ordered phase, $\phi_{\mathrm{o}}$, and the normalized boundary length, $l / l_{0}$, from 30 vesicles, and plotted them against $X_{\mathrm{PEG}}$ in Fig. 4b. With an increase in $m_{\mathrm{PEG}}$ for $0 \leq m_{\mathrm{PEG}} \leq 0.3, \phi_{\mathrm{o}}$ slightly increases. In contrast, for $m_{\mathrm{PEG}}>0.3, \phi_{\mathrm{o}}$ suddenly decreases below $\sim 0.1$, which is quite different from the monotonic increase in $\phi_{\mathrm{o}}$ in the Chol system (Fig. 2b). On the other hand, $l / l_{0}$ gradually increases from $\sim 5$ to $\sim 8$.

To explain this transition in the domain size, we focus on the amount of PEG-Chol contained in vesicles, which is expected to increase with an increase in $m_{\mathrm{PEG}}$, and to be saturated at the critical micelle concentration (CMC). With PEG-Chol in a PC membrane, the CMC was reported to be 0.4 for PEG600-Chol and 0.25 for PEG1000-Chol. ${ }^{30}$ In addition, a drastic change in permeability was observed above 0.3 for PEG900-Chol. ${ }^{31}$ Therefore, under our PEG600-Chol conditions, CMC is estimated to be $\sim 0.4$, which is slightly above our experimental value of $m_{\mathrm{PEG}}{ }^{*} \approx 0.3$ (Fig. 4). When we use longer PEG-Chol, such scattered domains were observed in vesicles with smaller $m_{\text {PEG }}$. Fig. 5a and $\mathrm{b}$ show scattered domains in vesicles composed of DPPC/DOPC $=1: 1$ plus 20\% PEG1000-Chol and PEG2000Chol, respectively. This agrees with the fact that $\mathrm{CMC}$ is decreased with an increase in the molecular weight of PEG.

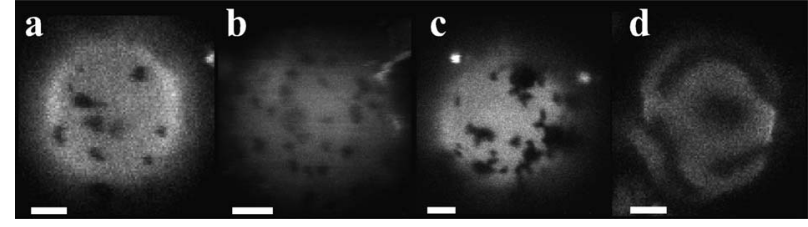

Fig. 5 Domain patterns on phase-separated vesicles containing (a) PEG1000-Chol 20\%, (b) PEG2000-Chol 20\%, (c) PEG600-Chol 20\%, and (d) PEG600-Chol 33\%. The bright and dark regions are DOPC-rich and DPPC-rich phases, respectively. Scale bar is $2 \mu \mathrm{m}$.

Therefore, it is most likely that when the value of $m_{\mathrm{PEG}}$ reaches the CMC, the increase in the free energy of PEG-Chol overcomes the cost of the line energy, and leads to a transition in the domain size.

On the other hand, in vesicles with low $m_{\mathrm{PEG}} \leq 0.3$, two types of domain patterns were observed, i.e., a mono-domain in the Chol plus PEG-Chol system (Fig. 3) and a network pattern in the PEG-Chol system (Fig. 4). Fig. 5c shows other examples of network patterns. After the samples were allowed to stand overnight, most of the domains were linked to their neighbors, although they did not completely merge into a larger monodomain. In addition, accompanied with the change of domain growth from nucleation-growth to a spinodal-decomposition mechanism, ${ }^{7}$ the characteristic pattern of spinodal-decomposition was also observed in vesicles with $m_{\text {chol }} \approx 0.3$ (Fig. $5 \mathrm{~d}$ ).

\section{Binary color imaging for network and scattered domains}

The presence of such noncircular domains has been reported in DPPC/DPPE GUVs with coexisting liquid-solid phases. ${ }^{32,33}$ Therefore, we examined whether the phase of DPPC-rich domains is a liquid or solid phase. For this experiment, we added two types of dyes, Rho-PE and NBD-PE. Rho-PE is localized in the Ld phase, whereas NBD-PE is relatively partitioned into Lo domains but not solid domains. ${ }^{14,34}$ Therefore, we can distinguish whether DPPC-rich domains are in a Lo phase or solid phase.

Fig. 6 shows binary color imaging of phase-separated vesicles, where red and green regions are Rho-PE and NBD-PE, respectively. In the case of solid domains in vesicles without Chol (Fig. 6a), NBD-PE was not localized in DPPC-rich domains. On the other hand, network domains (Fig. $6 \mathrm{~b}$ and $\mathrm{c}$ show the membrane surface and a cross-section, respectively) appeared green, which clearly shows that the domains are in the Lo phase, and not the solid phase. However, NBD-PE was excluded from the scattered domains (Fig. 6d), similar to the solid domains in Fig. 6a. Thus, the phase of the scattered domains is found to be close to the solid phase.

\section{Disruption by the laser irradiation and recovery}

To investigate the stability of the network domains, we irradiated part of the membrane with a pulsed laser, and examined whether or not the pattern is reproduced. Fig. 7 shows a time lapse of the domain patterns before and after laser irradiation. Before irradiation, small domains were connected to each other and formed a network pattern. Next, the white spot indicated by the arrow was irradiated with the laser $(80 \mathrm{~s})$. The 


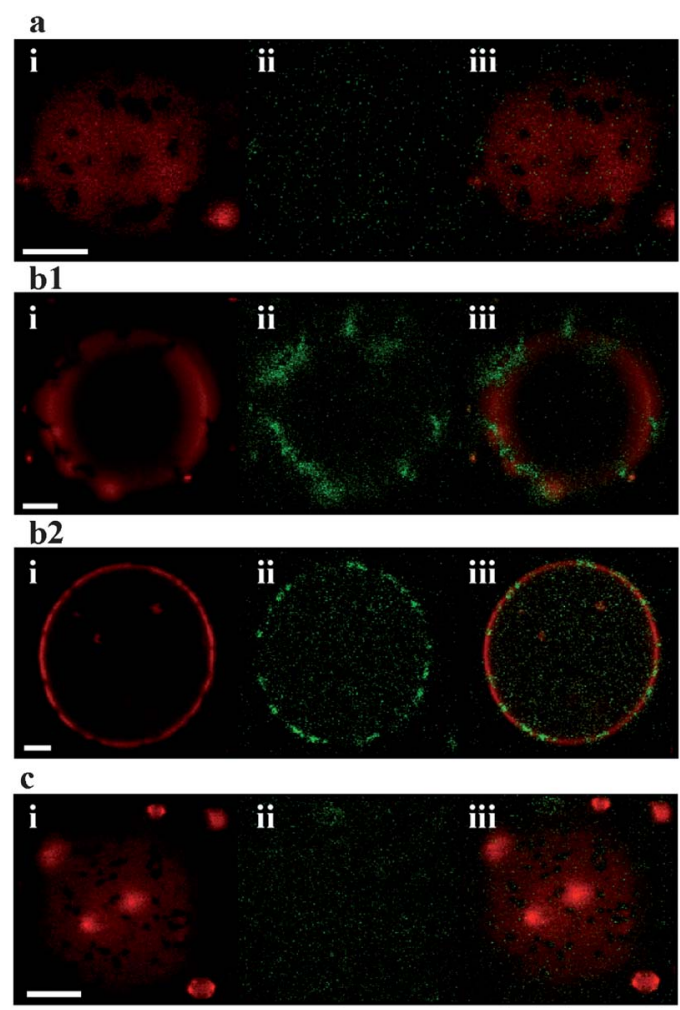

Fig. 6 Binary images of phase-separated vesicles composed of DPPC/ DOPC $=1: 1$ plus (a) Chol 0\%, (b1 and b2) PEG-Chol 30\%, and (c) PEG-Chol 40\%. These images focus on the membrane surface (a, b1 and c) or cross-section (b2). (i) Rho-PE (red) is localized in the DOPC-rich matrix. (ii) The partition of NBD-PE (green) into DPPC-rich domains is observed in (b) vesicles with PEG-Chol 30\%. Scale bar is $5 \mu \mathrm{m}$.

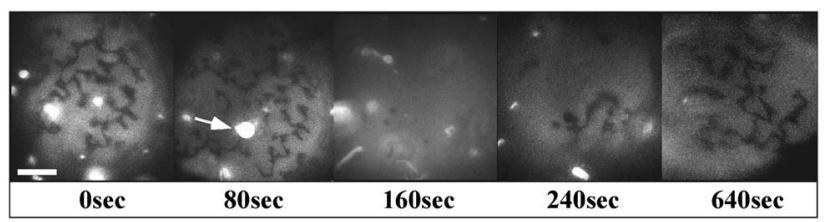

Fig. 7 Self-recovery of the network pattern after local heating. The vesicle is composed of DPPC/DOPC $=1: 1$ plus PEG-Chol 20\%. The part of the membrane indicated by a white circle and an arrow was irradiated with the laser for $\sim 80$ seconds from $80 \mathrm{~s}$ to $160 \mathrm{~s}$. Scale bar is $5 \mu \mathrm{m}$.

application of mechanical perturbation to the large domains was not effective, i.e., the domains could be moved by the laser but did not deform, and attached domains did not readily adhere to each other. However, thermal perturbation, or a heating effect, was observed over a longer time-scale. The network domains were gradually shrunken and scattered, and completely disappeared at $160 \mathrm{~s}$. When the laser was turned off, small domains appeared again and reproduced the network patterns. Since the characteristic size of the bead-like domains remained almost the same before and after laser irradiation, PEG-Chol may introduce spontaneous curvature to determine the pattern. ${ }^{22}$

\section{Discussion}

\section{Theoretical model}

As shown in Fig. 3, the transition from a mono-domain to small scattered domains was observed in four-component (DPPC/DOPC/Chol and PEG-Chol) vesicles with a high concentration of PEG-Chol. Under the experimental condition, the mole fraction of DPPC/DOPC/(Chol and PEG-Chol) was fixed to be $1: 1: 1$ and only the ratio of PEG-Chol to total Chol, $X_{\mathrm{PEG}}$, was changed. Therefore, we try to describe the domain size transition based on the free energy analysis as a function of $X_{\mathrm{PEG}}$ through the abstraction of the most important parameter among them.

For simplicity, we consider the phase-separated membrane without any shape deformation, where bending energy is ignored. We also ignore the entropic term, since we discuss the transition in the domain size, and not phase separation. In such a case, the balance between the repulsion of PEG-Chol molecules and the line tension at domain boundaries dominates the domain structure. Here we assume that there are $N$ domains of the Lo phase with the same radius $R$. When the total area of a vesicle is $S$, the total area of the Lo domains is denoted by $\phi_{\mathrm{o}} S$. The area fraction of the Lo domains, $\phi_{\mathrm{o}}$, depends on the mole ratio of PEG-Chol to total Chol $X_{\text {PEG }}$.

From the experimental result in Fig. 3, we use the relation

$$
\phi_{\mathrm{o}}=0.5-0.4 X_{\mathrm{PEG}}
$$

In addition, we use $m_{\mathrm{DPPC}}: m_{\mathrm{DOPC}}:\left(m_{\mathrm{PEG}}+m_{\text {chol }}\right)=1: 1: 1$. The cross-sectional areas of DPPC, DOPC and Chol have been reported to be $0.5,0.72$ and $0.37 \mathrm{~nm}^{2}$, respectively. ${ }^{27,28}$ Therefore, the area fraction of the total Chol is $\psi_{\mathrm{p}}+\psi_{\mathrm{c}} \approx 0.25$, where $\psi_{\mathrm{p}}$ and $\psi_{\mathrm{c}}$ are the area fractions of PEG-Chol and Chol. We obtain

$$
\psi_{\mathrm{p}}=X_{\mathrm{PEG}}\left(\psi_{\mathrm{p}}+\psi_{\mathrm{c}}\right)=0.25 X_{\mathrm{PEG}} .
$$

When the areas of the Lo and Ld phases are the same, Chol molecules become partitioned into a ratio of $m_{\text {chol_o }}: m_{\text {chol_d }}=$ $6: 4,{ }^{35}$ where $m_{\text {chol_o }}$ and $m_{\text {chol_d }}$ are the mole ratios of Chol in the Lo and Ld phases, respectively. Hence, it is natural that we assume that PEG-Chol molecules also become partitioned into the same ratio; that is

$$
n_{\mathrm{po}}: n_{\mathrm{pd}}=6 \phi_{\mathrm{o}} S: 4\left(1-\phi_{\mathrm{o}}\right) S,
$$

where $n_{\mathrm{po}}$ and $n_{\mathrm{pd}}$ are the numbers of PEG-Chol molecules in the Lo and Ld phases, respectively. The area fractions of PEG-Chol in the Lo and Ld phases are defined as $\psi_{\text {po }}$ and $\psi_{\mathrm{pd}}$ respectively. From eqn (2) and (3), and $\psi_{\mathrm{p}}=\psi_{\mathrm{po}}+\psi_{\mathrm{pd}}$, the area fraction of PEG-Chol in each phase can be expressed in terms of $X_{\text {PEG }}$ as

$$
\psi_{\mathrm{po}}=\frac{3 \phi_{\mathrm{o}}}{4\left(2+\phi_{\mathrm{o}}\right)} X_{\mathrm{PEG}}, \quad \psi_{\mathrm{pd}}=\frac{1-\phi_{\mathrm{o}}}{2\left(2+\phi_{\mathrm{o}}\right)} X_{\mathrm{PEG}}
$$

Since $N$ domains have the same radius $R$, we obtain $N \pi R^{2}=\phi_{\mathrm{o}} S$. The radius of the domains is described as

$$
R=\sqrt{\frac{\phi_{\mathrm{o}} S}{\pi N}} .
$$

PEG with a molecular weight 600 is considered to take a brush 
configuration, because the polymer length $(\sim 0.6 \mathrm{~nm})$ is smaller than the persistence length of PEG $(\sim 0.9 \mathrm{~nm}) .{ }^{26}$ From scaling theory, the free energy per polymer grafted on the lipid membrane derived from the interaction between polymers is given by ${ }^{10,36-39}$

$$
f_{\mathrm{p}}=k_{\mathrm{B}} T m a_{\mathrm{m}}^{5 / 3} d^{5 / 6},
$$

where $m$ is the degree of polymerization, $a_{\mathrm{m}}$ is the monomer size, $d$ is the density of PEG-Chol, $k_{\mathrm{B}}$ is the Boltzmann constant, and $T$ is the temperature. This energy is also obtained from meanfield theory. ${ }^{40,41}$ Although there are some differences between scaling theory and mean-field theory (e.g., the constant premultiplying factor and the dependence on the polymer density), they do not affect the phase behavior qualitatively. For simplicity, we assume that PEG-Chol molecules disperse uniformly in the Lo and Ld phases.

Here, we consider that PEG-Chol near the domain boundary feels a different energy from that in the center of the Lo or Ld phase, due to the difference in the density of PEGChol between the Lo and Ld phases. As illustrated in Fig. 8, PEG-Chol in Lo domains feels free energy $f_{\text {po }}$ per molecule (region A). On the other hand, the energy of PEG-Chol near the domain boundary (region B) is reduced compared with that of PEG-Chol at the center of the domain, since PEG-Chol near the domain boundary effectively feels a lower density. In contrast, PEG-Chol in the Ld phase near the domain boundary (region C) feels higher energy than that in the center of Ld phase (region D). We introduce the phenomenological factor $\gamma$ to briefly express an increase and a decrease in free energy in region $\mathrm{B}$ and $\mathrm{C}$.

Regions B and C lie between the domain boundary and the lines are at distance $\delta$ inward and outward, respectively, from the domain boundary. Since the energy induced by PEG-Chol is expressed as the sum of the contributions from these four regions, the energy attributable to PEG-Chol per unit area is given by
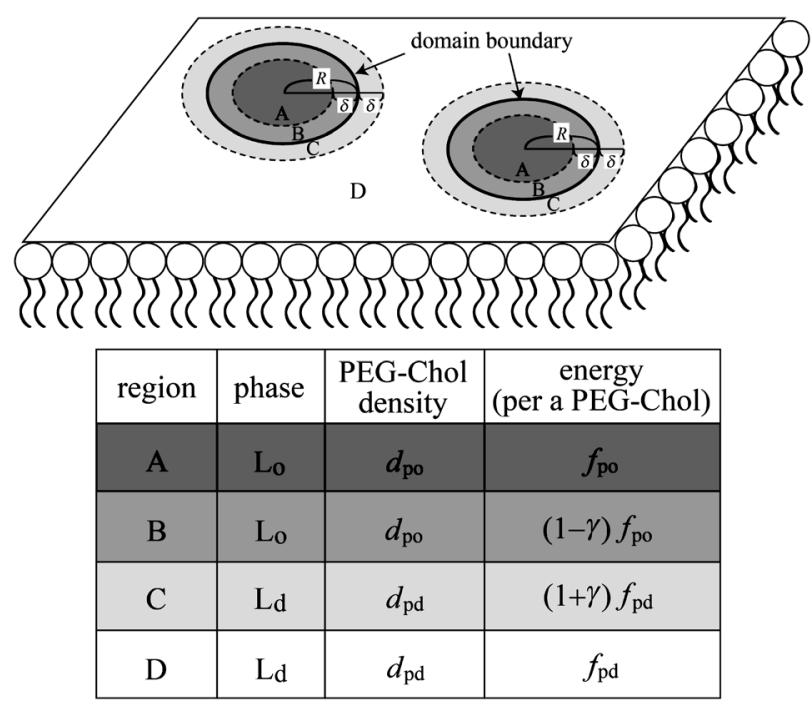

Fig. 8 Schematic illustration of the phase-separated membrane. Phases, PEG-Chol density and energy per PEG-Chol molecule in each region are shown.

$$
\begin{gathered}
F_{\mathrm{p}}=\frac{\pi(R-\delta)^{2}}{\pi R^{2}} \frac{n_{\mathrm{po}}}{S} f_{\mathrm{po}}+\left[1-\frac{\pi(R-\delta)^{2}}{\pi R^{2}}\right] \frac{n_{\mathrm{po}}}{S}(1-\gamma) f_{\mathrm{po}} \\
+\frac{N\left[\pi(R+\delta)^{2}-\pi R^{2}\right]}{\left(1-\phi_{\mathrm{o}}\right) S} \frac{n_{\mathrm{pd}}}{S}(1+\gamma) f_{\mathrm{pd}} \\
+\left[1-\frac{N\left[\pi(R+\delta)^{2}-\pi R^{2}\right]}{\left(1-\phi_{\mathrm{o}}\right) S}\right] \frac{n_{\mathrm{pd}}}{S} f_{\mathrm{pd}}
\end{gathered}
$$

where $f_{\text {po }}$ and $f_{\text {pd }}$ are the energies of a PEG-Chol molecule in regions $\mathrm{A}$ and $\mathrm{D}$, and $(1-\gamma) f_{\mathrm{po}}$ and $(1+\gamma) f_{\mathrm{pd}}$ are the energies of a PEG-Chol in regions $\mathrm{B}$ and $\mathrm{C}$, respectively. Here, we substitute the relations

$$
d_{\mathrm{po}}=\frac{n_{\mathrm{po}}}{\phi_{\mathrm{o}} S}=\frac{\psi_{\mathrm{po}}}{A_{\mathrm{p}} \phi_{\mathrm{o}}}, \quad d_{\mathrm{pd}}=\frac{n_{\mathrm{pd}}}{\left(1-\phi_{\mathrm{o}}\right) S}=\frac{\psi_{\mathrm{pd}}}{A_{\mathrm{p}}\left(1-\phi_{\mathrm{o}}\right)}
$$

into eqn (7), where $A_{\mathrm{p}}$ is the cross-sectional area of a PEG-Chol molecule. Thus, we obtain the following formula,

$$
\begin{aligned}
F_{\mathrm{p}} & =k_{\mathrm{B}} T\left\{\left[1-\frac{\gamma(2 R-\delta) \delta}{R^{2}}\right] m a_{\mathrm{m}}^{5 / 3} A_{\mathrm{p}}^{-11 / 6} \phi_{\mathrm{o}}^{-5 / 6} \psi_{\mathrm{po}}^{11 / 6}\right. \\
& \left.+\left[1+\frac{\gamma \pi(2 R+\delta) \delta N}{\left(1-\phi_{\mathrm{o}}\right) S}\right] m a_{\mathrm{m}}^{5 / 3} A_{\mathrm{p}}^{-11 / 6}\left(1-\phi_{\mathrm{o}}\right)^{-5 / 6} \psi_{\mathrm{pd}}^{11 / 6}\right\} .
\end{aligned}
$$

From eqn (1), (4), and (5), the free energy induced by PEGChol, $F_{\mathrm{p}}$, can be expressed as a function of the number of domains $N$ and the ratio of PEG-Chol to total Chol, $X_{\mathrm{PEG}}$. Since we fix the lipid and PEG-Chol concentrations in the Lo and Ld phases, the concentration gradient across the domain boundary is constant. Therefore, the line energy depends only on the length of the domain boundary. The line energy at the domain boundary per unit area $F_{1}$ is expressed as

$$
F_{1}=\frac{2 \pi R N}{S} f_{1}
$$

where $f_{1}$ is the line tension.

To explain the domain size transition theoretically, we calculate the total free energy $F_{\mathrm{p}}+F_{1}$ as a function of $X_{\mathrm{PEG}}, f_{1}$ and $\gamma$. In the calculation, we use $S=314 \mu \mathrm{m}^{2}$ (the radius of a vesicle is $5 \mu \mathrm{m}), \delta=10 \mathrm{~nm}, m=13, a_{\mathrm{m}}=0.4 \mathrm{~nm}, A_{\mathrm{p}}=0.4$ $\mathrm{nm}^{2}$, and $T=300 \mathrm{~K}^{42,43}$ The effective densities of PEG-Chol near the boundary are given by $(1-\gamma)^{6 / 5} d_{\mathrm{po}}$ and $(1+\gamma)^{6 / 5} d_{\mathrm{pd}}$ in regions $B$ and $C$, respectively. Because the densities should follow the relation: $d_{\mathrm{po}}$ (region A) $>(1-\gamma)^{6 / 5} d_{\mathrm{po}}$ (region $\left.\mathrm{B}\right)>(1$ $+\gamma)^{6 / 5} d_{\mathrm{pd}}\left(\right.$ region C) $>d_{\mathrm{pd}}($ region D), we obtain $0<\gamma<0.167$ using eqn (3) and (8).

First, we fix the parameters $\gamma=0.1$ and $f_{1}=3.0 \mathrm{pN}$, and obtain the dependence of $X_{\mathrm{PEG}}$ on the length of the domain boundary $l$ (dot-dashed line in Fig. 9). Fig. 9 represents the normalized domain boundary length, $l / l_{0}$, where $l_{0}$ is the domain boundary length of a mono-domain, i.e., $N=1$. When there are $N$ domains, the total domain boundary length $l$ is denoted by $l=2\left(\pi \phi_{\mathrm{o}} S N\right)^{1 / 2}$. Since we can obtain $l_{0}=2\left(\pi \phi_{\mathrm{o}} S\right)^{1 / 2}$, the normalized domain boundary length becomes $l / l_{0}=\sqrt{N}$. It should be noted that the radius of a domain $R$ must be larger than $\delta$. From eqn (5), the number of domain is $N=10^{5}$ and the domain boundary length is $l / l_{0}=316$ when $R$ and $\delta$ are both $10 \mathrm{~nm}$ at $X_{\mathrm{PEG}}=100 \%$. As 


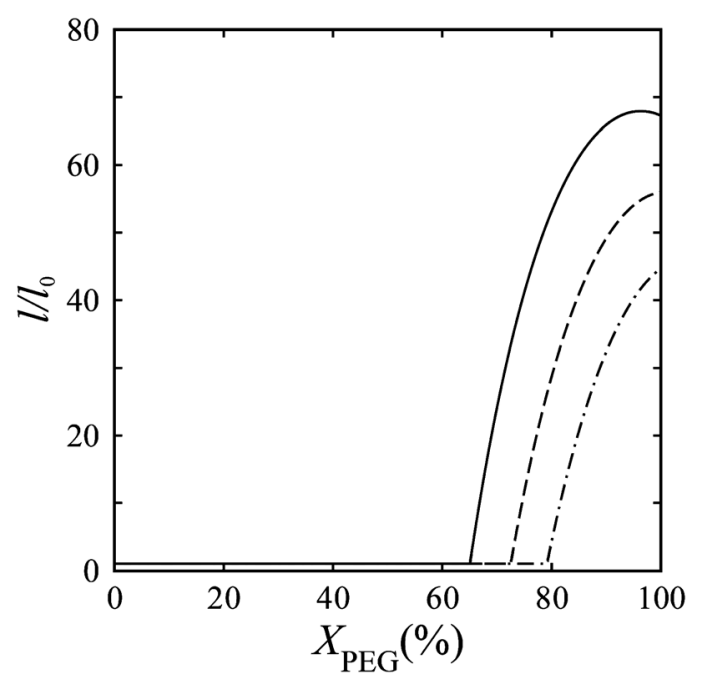

Fig. 9 The change in the length of the domain boundary as a function of $X_{\mathrm{PEG}}$ for $\gamma=0.10$. The solid, dashed and dot-dashed lines represent $f_{1}=$ $2.0,2.5$, and $3.0 \mathrm{pN}$, respectively.

shown in Fig. 9, the calculated value is much smaller than $l / l_{0}=$ 316 , and the above condition is always fulfilled throughout this model.

When $X_{\mathrm{PEG}}$ is small enough, line energy dominates the phase behaviour and the mono-domain $\left(I / l_{0}=1\right)$ appears. As $X_{\mathrm{PEG}}$ becomes larger, the interaction energy between polymers becomes higher. Because the PEG-Chol densities in Lo and Ld phases are increased. While the line energy is decreased with an increase of $X_{\mathrm{PEG}}$ from eqn (1). Hence, when $X_{\mathrm{PEG}}$ is increased, the point where the interaction energy between polymers overcomes the line energy is reached. The transition from the monodomain $\left(I / l_{0}=1\right)$ to scattered domains $\left(I / l_{0}>1\right)$ is occurred at this point. As shown in Fig. 9, such transition points appear at $X_{\mathrm{PEG}}$ $=65 \%\left(f_{1}=2.0 \mathrm{pN}\right), 73 \%\left(f_{1}=2.5 \mathrm{pN}\right)$, and $79 \%\left(f_{1}=3.0 \mathrm{pN}\right)$.

On the other hand, the total areas of region $\mathrm{B}$ and $\mathrm{C}$ are expressed by $\pi N R^{2}-\pi N(R-\delta)^{2}$ and $\pi N(R+\delta)^{2}-\pi N R^{2}$, respectively. The area difference between region $\mathrm{B}$ and $\mathrm{C}$ is $\mid 2 \phi_{\mathrm{O}} S$ $(\delta / R)^{2} \mid$. As the domain size $R$ becomes smaller, this area difference becomes remarkable. This means that the ratio of the free energy loss in region $\mathrm{C}$ relatively becomes larger when the domain size is small enough. Since the domain size becomes smaller with increasing $X_{\mathrm{PEG}}$, it is energetically unfavourable to increase the number of domains and decrease the domain size for large $X_{\mathrm{PEG}}$. Therefore, with an increase of $X_{\mathrm{PEG}}, l / l_{0}$ increases and again decreases to minimize the energy. For $f_{1}=2.0 \mathrm{pN}$, we can find this point as a peak around $X_{\mathrm{PEG}}=95 \%$ (solid line in Fig. 9).

Second, we analyze the dependence of line tension $f_{1}$ on the relation between $X_{\mathrm{PEG}}$ and $l / l_{0}$ using different line tension $f_{1}=$ $2.0,2.5,3.0 \mathrm{pN}$ (Fig. 9). The critical $X_{\mathrm{PEG}}$ at the transition point is increased from $\sim 60 \%$ to $\sim 80 \%$ with increasing $f_{1}$ from $2 \mathrm{pN}$ to $3 \mathrm{pN}$. These values of line tension have the same magnitude as those in previous experimental reports. ${ }^{15,29}$

In addition, the slope of the change in $l / l_{0}$ at the transition point is quite large. This means that the transition from a monodomain to scattered domains occurs sharply. As shown in Fig. 3,

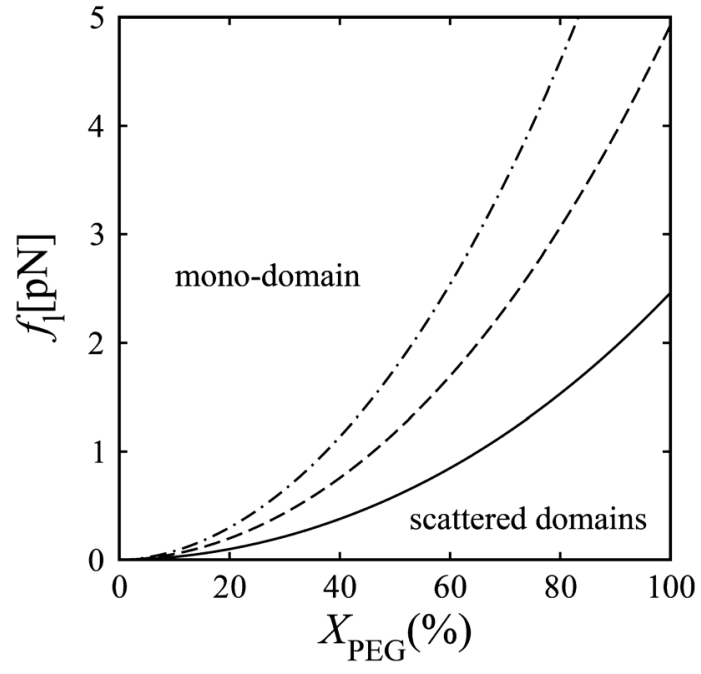

Fig. 10 Phase diagram calculated as a function of $X_{\mathrm{PEG}}$ and the line tension at the domain boundary $f_{1}$. The solid, dashed, dot-dashed lines represent $\gamma=0.05,0.10$, and 0.15 , respectively.

there is only one domain for $X_{\mathrm{PEG}} \%=60$, while the number of domains rapidly increases for $X_{\mathrm{PEG}} \%=80$. This behaviour of the transition is also consistent with that in the experiment.

As for the domain boundary length $l / l_{0}$, the calculated domain boundary length tends to be larger than the experimental values $\left(l / l_{0}<9\right)$. This discrepancy may be due to the resolution of the fluorescent microscope $(\sim 1 \mu \mathrm{m})$. For example, in the case of domains with $2 R=1 \mu \mathrm{m}$, the domain boundary length is $l / l_{0}=$ 8.4 for $X_{\mathrm{PEG}}=80 \%$. Even if the domains smaller than $1 \mu \mathrm{m}$ exist, it is difficult to measure such boundary lengths of small domains by a microscope. Hence, the domain boundary length obtained from the experiment becomes smaller.

Furthermore, the critical $X_{\mathrm{PEG}}$ at the transition point is strongly dependent on line tension, $f_{1}$ and also $\gamma$. The calculated phase diagram as a function of $X_{\mathrm{PEG}}$ and $f_{1}$ is shown in Fig. 10. Using eqn (1) and (5), the phase boundaries are obtained by minimizing the total free energy $F_{\mathrm{p}}+F_{1}$ with respect to the number of domain $N$ for $\gamma=0.05,0.10$, and 0.15 . Generally, the line tension and the parameter $\gamma$ are dependent on the lipid composition and temperature. ${ }^{15,29,44}$ Therefore, this transition from global- to micro-segregation would be triggered depending on the type of bulky-head lipids, the lipid composition and the temperature, also in cellular membranes.

\section{Conclusions}

We investigated the effects of bulky-head lipids on phase separation using a giant vesicle composed of DPPC/DOPC $=1: 1$ plus different mole fractions of Chol and/or PEG-Chol. Basic ternary vesicles composed of DPPC, DOPC and Chol show phase separation into two liquid phases, i.e., liquid-ordered and liquid-disordered phases, and form a mono-domain to minimize the line energy at domain boundaries. When Chol in the ternary vesicles is replaced with PEG-Chol, the size of the mono-domain is decreased. This indicates that PEG-Chol is excluded from the DPPC-rich ordered domain. Above the critical concentration of PEG-Chol, near the critical micelle concentration of PEG-Chol, 
the domain structure is transformed from a mono-domain to small scattered domains. This change in the domain size can be explained theoretically by comparing the repulsion between PEG-Chol and the cost of the line energy at the domain boundaries. In addition, network domains, where small domains are connected to each other, were observed as an intermediate state. We examined the stability of the network pattern by local heating using a focused laser, and confirmed that it exhibited selfrecovery. Based on the present results, bulky-head lipids are expected to stabilize nano-domain structures even in living cells.

We thank Hiroshi Noguchi, Takashi Taniguchi and Takeaki Araki for their useful discussions. This work was supported by the Japan Society for the Promotion of Science (JSPS) (Grant-inAid for Scientific Research (A); no. 23240044) and by the Ministry of Education, Culture, Sports, Science and Technology (Scientific Research on Innovative Areas; no. 23106712). M.Y. and N.S. are grateful for support from JSPS (Grants-in-Aid for JSPS Fellows; no. 22-940 (M.Y.), no. 23-8690 (N.S.)).

\section{References}

1 K. Simons and E. Ikonen, Nature, 1997, 387, 569-572.

2 K. Jacobson, O. G. Mouritsen and R. G. Anderson, Nat. Cell Biol., 2007, 9, 7-14.

3 L. J. Pike, J. Lipid Res., 2006, 47, 1597-1598.

4 Y. M. Umemura, M. Vrljic, S. Y. Nishimura, T. K. Fujiwara, K. G. N. Suzuki and A. Kusumi, Biophys. J., 2008, 95, 435-450.

5 C. Eggeling, C. Ringemann, R. Medda, G. Schwarzmann, K. Sandhoff, S. Polyakova, V. N. Belov, B. Hein, C. von Middendorff, A. Schonle and S. W. Hell, Nature, 2009, 457, 11591162.

6 S. L. Veatch and S. L. Keller, Phys. Rev. Lett., 2002, 89, 268101.

7 S. L. Veatch and S. L. Keller, Biophys. J., 2003, 85, 3074-3083.

8 S. L. Veatch and S. L. Keller, Biochim. Biophys. Acta, Gen. Subj., 2005, 1746, 172-185.

9 S. L. Veatch, O. Soubias, S. L. Keller and K. Gawrisch, Proc. Natl. Acad. Sci. U. S. A., 2007, 104, 17650-17655.

10 M. Yanagisawa, M. Imai, T. Masui, S. Komura and T. Ohta, Biophys. J., 2007, 92, 115-125.

11 M. Yanagisawa, M. Imai and T. Taniguchi, Phys. Rev. Lett., 2008, 100, 148102.

12 M. Yanagisawa, M. Imai and T. Taniguchi, Phys. Rev. E: Stat., Nonlinear, Soft Matter Phys., 2010, 82, 051928.

13 G. W. Feigenson and J. T. Buboltz, Biophys. J., 2001, 80, 2775-2788.

14 N. Shimokawa, M. Hishida, H. Seto and K. Yoshikawa, Chem. Phys. Lett., 2010, 496, 59-63.

15 T. Baumgart, S. T. Hess and W. W. Webb, Nature, 2003, 425, 821824.
16 P. M. Rudd, B. P. Morgan, M. R. Wormald, D. J. Harvey, C. W. vandenBerg, S. J. Davis, M. A. J. Ferguson and R. A. Dwek, J. Biol. Chem., 1997, 272, 7229-7244.

17 G. Montesano, R. Bartucci, S. Belsito, D. Marsh and L. Sportelli, Biophys. J., 2001, 80, 1372-1383.

18 F. M. Thakkar and K. G. Ayappa, J. Phys. Chem. B, 2010, 114, 2738 2748.

19 S. Belsito, R. Bartucci, G. Montesano, D. Marsh and L. Sportelli, Biophys. J., 2000, 78, 1420-1430.

20 D. Marsh, Biophys. J., 2001, 81, 2154-2162.

21 T. Masui, M. Imai, K. Nakaya and T. Taniguchi, J. Chem. Phys., 2006, 124, 74904.

22 H. E. Warriner, S. H. Idziak, N. L. Slack, P. Davidson and C. R. Safinya, Science, 1996, 271, 969-973.

23 T. Taniguchi, Phys. Rev. Lett., 1996, 76, 4444-4447.

24 R. Lipowsky and R. Dimova, J. Phys.: Condens. Matter, 2003, 15, S31-S45.

25 B. Cabane and R. Duplessix, J. Phys. (Paris), 1993, 51, 1313.

26 S. Kawaguchi, G. Imai, J. Suzuki, A. Miyahara and T. Kitano, Polymer, 1997, 38, 2885-2891.

27 J. F. Nagle and S. Tristram-Nagle, Biochim. Biophys. Acta, Gen. Subj., 2000, 1469, 159-195.

28 H. S. Shieh, L. G. Hoard and C. E. Nordman, Acta Crystallogr., Sect. B: Struct. Crystallogr. Cryst. Chem., 1981, 37, 1538-1543.

29 A. Tian, C. Johnson, W. Wang and T. Baumgart, Phys. Rev. Lett., 2007, 98, 208102.

30 J. Janzen, X. Song and D. E. Brooks, Biophys. J., 1996, 70, 313-320.

31 S. Spiwongsitanont and M. Ueno, Chem. Pharm. Bull., 2002, 50, $1238-1244$.

32 P. A. Beales, V. D. Gordon, Z. J. Zhao, S. U. Egelhaaf and W. C. K. Poon, J. Phys.: Condens. Matter, 2005, 17, S3341-S3346.

33 V. D. Gordon, P. A. Beales, Z. Zhao, C. Blake, F. C. MacKintosh, P. D. Olmsted, M. E. Cates, S. U. Egelhaaf and W. C. K. Poon, J. Phys.: Condens. Matter, 2006, 18, L415-L420.

34 J. Yuan, S. M. Hira, G. F. Strouse and L. S. Hirst, J. Am. Chem. Soc., 2008, 130, 2067-2072.

35 S. L. Veatch, I. V. Polozov, K. Gawrisch and S. L. Keller, Biophys. J., 2004, 86, 2910-2922.

36 P. G. de Gennes, Macromolecules, 1980, 13, 1069.

37 Scaling Concepts in Polymer Physics, ed. P. G. D. Gennes, Connel Univ. Press, London, 1979.

38 L. G. Alexander, J. Phys., 1977, 38, 983-987.

39 M. Daoud and P. G. D. Gennes, J. Phys., 1977, 38, 85-93.

40 S. T. Milner, T. A. Witten and M. E. Cates, Macromolecules, 1988, 21, 2610-2619.

41 S. T. Milner, T. A. Witten and M. E. Cates, Europhys. Lett., 1988, 5, 413-418.

42 K. Hristova and D. Needham, Macromolecules, 1995, 28, 9911002.

43 E. Evans, D. J. Klingenberg, W. Rawicz and F. Szoka, Langmuir, 1996, 12, 3031-3037.

44 I. Levental, M. Grzybek and K. Simons, Proc. Natl. Acad. Sci. U. S. A., DOI: 10.1073/pnas. 1105996108 . 